\title{
Preface
}

\section{Structures of the Anterolateral Knee: Why All the Confusion?}

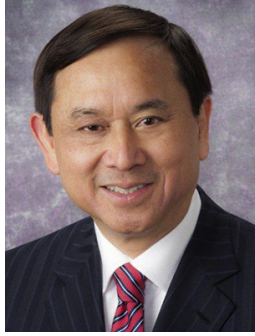

Freddie H. Fu, MD

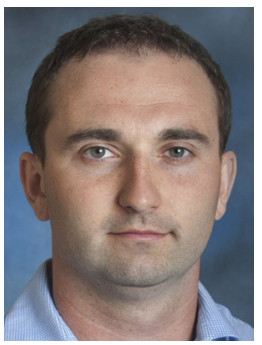

Marcin Kowalczuk, MD, FRCSC Editors

Over the last two decades we have seen a shift in anterior cruciate ligament (ACL) reconstruction that focuses on restoration of the native anatomy. Despite this anatomic approach, a subset of patients continue to experience anterolateral rotatory instability. This persistent instability suggests that pieces to the puzzle that is complex knee instability are still missing. Continued efforts to improve clinical outcomes have led to the recent resurgence of interest in the anatomic structures of the anterolateral knee, termed the anterolateral complex (ALC). Most notably this has yielded a significant body of literature regarding the anterolateral ligament (ALL). This renewed interest has led to healthy academic debate as well as significant confusion.

The confusion stems from the inherent complexity of the lateral knee anatomy. Historically the structures of the ALC and ALL have gone by many names, which has contributed to inconsistencies of anatomic nomenclature. ${ }^{1}$ Many of these inconsistencies are due to differences in dissection technique and the variable use of embalmed versus fresh frozen specimens in study protocols. The process of tissue embalming changes tissue consistency and blurs important planes of dissection. This is of paramount importance when discerning the difference between capsular thickenings and discrete ligamentous tissues. The aforementioned factors have all contributed to the confusion regarding the anatomic structures of the ALC and ALL. A lack of consensus on anatomic nomenclature naturally translates into a poor understanding of function, which can be seen in the variable results across biomechanical studies of the ALC and ALL.

In an effort to correct or prevent residual anterolateral knee instability, orthopedic surgeons have increasingly turned to lateral tenodesis and reconstruction procedures. These lateral procedures, while potentially decreasing rotatory instability, can also lead to overconstraint, increased external tibial rotation, and greater contact stresses in the lateral compartment. ${ }^{2,3}$ Although the body of literature regarding the ALC and ALL 
continues to grow, it is chiefly limited to cadaveric studies and case series. High-level studies in the form of randomized clinical trials are on the horizon, but for the time being augmentation of $A C L$ reconstruction with lateral procedures should be approached with caution and considered in each patient individually. Surgical indications remain unclear, and undue long-term harm remains a distinct possibility.

The puzzle that is complex knee instability continues to challenge clinicians. It is important to remember that the pivot shift phenomenon is multifactorial, with bone morphology, ligamentous laxity, meniscal tissues, and the posterolateral and anterolateral structures all playing contributory roles. ${ }^{4}$ The anterolateral knee structures are a critical piece of the puzzle but have also been the source of considerable confusion. In an effort to provide clarity, we hope that this special issue of Clinics in Sports Medicine authored by an international panel of experts will provide an open arena for the exchange of knowledge and critical appraisal of the literature. With an improved understanding of anatomy, biomechanic function, and surgical indications, improved patient outcomes in this challenging patient population can be achieved.

\author{
Freddie H. Fu, MD \\ Department of Orthopaedic Surgery \\ University of Pittsburgh \\ UPMC Center for Sports Medicine \\ 3200 South Water Street \\ Pittsburgh, PA 15203, USA \\ Marcin Kowalczuk, MD, FRCSC \\ Department of Orthopaedic Surgery \\ University of Pittsburgh \\ UPMC Center for Sports Medicine \\ 3200 South Water Street \\ Pittsburgh, PA 15203, USA \\ E-mail addresses: \\ ffu@upmc.edu (F.H. Fu) \\ kowalczukm@upmc.edu (M. Kowalczuk)
}

\title{
REFERENCES
}

1. Cavaignac E, Ancelin D, Chiron P, et al. Historical perspective on the "discovery" of the anterolateral ligament of the knee. Knee Surg Sports Traumatol Arthrosc 2017;25(4):991-6.

2. Inderhaug E, Stephen JM, Williams A, et al. Biomechanical comparison of anterolateral procedures combined with anterior cruciate ligament reconstruction. Am J Sports Med 2017;45(2):347-54.

3. Slette EL, Mikula JD, Schon JM, et al. Biomechanical results of lateral extra-articular tenodesis procedures of the knee: a systematic review. Arthroscopy 2016; 32(12):2592-611.

4. Fu FH, Herbst E. Editorial commentary: the pivot-shift phenomenon is multifactorial. Arthroscopy 2016;32(6):1063-4. 